\title{
M.
}

\section{Effects of flurbiprofen axetil on postoperative serum IL-2 and IL-6 levels in patients with colorectal cancer}

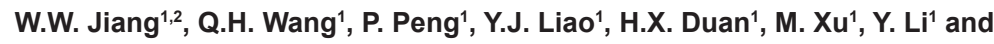 \\ P.B. Zhang ${ }^{2}$ \\ 'Department of Anesthesiology II, Affiliated Zhongshan Hospital of Dalian University, \\ Dalian, China \\ ${ }^{2}$ Department of Anesthesiology, \\ Second Affiliated Hospital of Xi'an Jiaotong University School of Medicine, \\ Xi'an, Shanxi, China \\ Corresponding author: P.B. Zhang \\ E-mail: pengbozhangdoc@yeah.net \\ Genet. Mol. Res. 14 (4): 16469-16475 (2015) \\ Received July 15, 2015 \\ Accepted September 20, 2015 \\ Published December 9, 2015 \\ DOI http://dx.doi.org/10.4238/2015.December.9.18
}

ABSTRACT. We explored the effects of flurbiprofen axetil on interleukin (IL)-2 and IL-6 levels in postoperative patients with colorectal cancer. A total of 120 patients (American Society of Anesthesiologists I and II) scheduled to undergo colorectal cancer surgery were randomly divided into 3 groups $(\mathrm{N}=40$ in each group): flurbiprofen axetil group (group $\mathrm{F}$ ), morphine group (group M), and tramadol group (group T). Group M received $0.1 \mathrm{mg} / \mathrm{kg}$ morphine, group $\mathrm{T}$ received $1.5 \mathrm{mg} / \mathrm{kg}$ tramadol, and group $\mathrm{F}$ received $1.5 \mathrm{mg} / \mathrm{kg}$ flurbiprofen axetil. Patients in the 3 groups were administered treatments through intravenous injection 10 min before surgery. Serum IL-2 and IL-6 levels were detected. Postoperative adverse reactions were recorded, such as nausea, vomiting, and pruritus. The serum IL-6 level of the 3 groups increased $3 \mathrm{~h}$ after surgery. Compared with group M, IL-6 level was higher in group $\mathrm{T}$ and group $\mathrm{F}$ at 1 day after the surgery, and the differences between group $M$ and the other groups were significant 
$(P<0.05)$. Moreover, the incidence of adverse reactions was significantly different among 3 groups $(P<0.05)$. Flurbiprofen axetil promoted the secretion of IL-2 and inhibited IL-6; additionally, flurbiprofen axetil may have a lower incidence of adverse reactions compared to other treatments.

Key words: Cytokines; Flurbiprofen axetil; Immunosuppressive; Morphine; Tramadol

\section{INTRODUCTION}

Perioperative immunosuppression has gained increasing attention. Anesthesiological drugs must be chosen cautiously for immunocompromised tumor patients whose immune function is lower than that of healthy people. Surgery, anesthesiological drug use, and complex changes in endocrine metabolism may affect defense reactions or the immune system. These drugs activate the hypothalamic-pituitary-adrenal axis or sympathy-adrenal medulla system (Gibson, 1996), altering immune system function and increasing opportunistic infection and tumor progression. Generally, patients should be awoken immediately after surgery, but some patients suffer from brain disorder, such confusion, hypersomnia, and restlessness. Although restlessness is transient, it leads to severe complications, such as accidental injury (Atkins, 2002; Roy et al., 2011). Morphine and tramadol are widely used to prevent postoperative restlessness in clinical application. Although morphine is generally acknowledged as an immunosuppressant, long-term use and large-doses of morphine will inhibit the proliferation of natural killer cells and $\mathrm{T}$ and $\mathrm{B}$ lymphocytes, as well as reduce the levels of cytokines such as interleukin (IL)-2, IL-4, and IL-6 (Pahl, 1999). Tramadol is an atypical opioid used primarily to treat moderate-severe pain, both acute and chronic. The most common adverse effects of tramadol include dizziness, dry mouth, nausea, indigestion, abdominal pain, vertigo, vomiting, constipation, drowsiness, and headache (Mejjad et al., 2011). Flurbiprofen axetil is a new non-steroidal anti-inflammatory and analgesic drug, which avoids topically stimulating the digestive tract through intravenous injection. Moreover, this drug shows high affinity to inflammatory tissues because it uses a lipid microspheres system. Furthermore, flurbiprofen axetil does not cause development of respiratory depression. In this study, we compared the differences in postoperative serum IL-2 and IL-6 levels in patients with colorectal cancer after treatment with flurbiprofen axetil, morphine, and tramadol. Our results will be useful when choosing analgesic drug in perioperative periods (Fujii and Itakura, 2008, 2009).

\section{MATERIAL AND METHODS}

\section{General data}

A total of 120 patients (American Society of Anesthesiologists I-II), aged from 31-65 years weighing 43-80 $\mathrm{kg}$, who had undergone elective colorectal cancer were included in the study. Patients were excluded if they had allergies to non-steroidal anti-inflammatory drugs, renal dysfunction, or coagulation disorders and had used non-steroidal anti-inflammatory drugs, narcotic analgesic, enoxacin, lomefloxacin, and norfloxacin within $24 \mathrm{~h}$ before surgery. This study was conducted in accordance with the Declaration of Helsinki. This study was conducted with approval from the Ethics Committee of Affiliated Zhongshan Hospital of Dalian University. Written informed consent was obtained from all participants. 


\section{Groups}

The 120 patients were randomly divided into 3 groups, with each group containing 40 patients. The morphine group (group $M$ ) received intravenous injection of $0.1 \mathrm{mg} / \mathrm{kg}$ morphine $10 \mathrm{~min}$ before surgery, the tramadol group (group T) received intravenous injection of $1.5 \mathrm{mg} / \mathrm{kg}$ tramadol $10 \mathrm{~min}$ before surgery, and the flurbiprofen axetil group (group F) received intravenous injection of $1.5 \mathrm{mg} / \mathrm{kg}$ flurbiprofen axetil $10 \mathrm{~min}$ before surgery. Postoperative adverse effects were recorded, including nausea, vomiting, and pruritus.

\section{Anesthesia and sample collection}

Patients in all groups received intravenous anesthesia, including $0.06 \mathrm{mg} / \mathrm{kg}$ midazolam, $5 \mu \mathrm{g} / \mathrm{kg}$ fentanyl, $1 \mathrm{mg} / \mathrm{kg}$ propofol, and $1.5 \mathrm{mg} / \mathrm{kg}$ vecuronium. Tracheal cannula was followed by anesthesia. Next, sevoflurane was inhaled to maintain anesthesia and the minimum alveolar concentration was approximately 2.1. Additionally, cisatracurium was injected discontinuously to maintain muscular fasciculation during surgery. After surgery, patients were transferred to the recovery room. If patients were awake and had normal physical indices, for example, tidal volume $>8 \mathrm{mg} / \mathrm{kg}$ and $\mathrm{SPO}_{2}>95 \%$, the tracheal catheter was removed. Peripheral venous blood samples were drawn preoperatively $\left(T_{0}\right)$ and at $3 \mathrm{~h}, 1$ day, and 3 days postoperatively to detect the plasma level of IL-2 and IL-6 using an enzyme-linked immunosorbent assay (ELISA). The ELISA kit was purchased from Beijing KeMei Bio-Tech, Ltd. (Beijing, China). All samples were stored at $-20^{\circ} \mathrm{C}$ after centrifugation.

\section{Statistical analysis}

Statistical analysis was performed using SPSS 13.0 software (SPSS, Inc., Chicago, IL, USA). Measured data were presented as the mean \pm SD. Students $t$-test was used to compare data between groups. The paired $t$-test was used to compare data in the same group. Count data was compared using chi-square test. A P value $<0.05$ denoted a significant statistical difference.

\section{RESULTS}

\section{General data}

There were no significant differences among the 3 groups in terms of age, body weight, and surgery duration ( $P>0.05$, Table 1$)$.

Table 1. General data of three groups (means $\pm \mathrm{SD}, \mathrm{N}=40)$.
\begin{tabular}{lccrr}
\hline Group & Counts & Age/years & Body weight/kg & Duration of surgery/min \\
\hline Group M & 40 & $48.4 \pm 8.9$ & $61.23 \pm 8.3$ & $91.23 \pm 30.4$ \\
Group T & 40 & $49.5 \pm 9.2$ & $66.3 \pm 4.5$ & $89.23 \pm 23.2$ \\
Group F & 40 & $47.1 \pm 5.9$ & $65.8 \pm 6.6$ & $92.5 \pm 34.2$ \\
\hline
\end{tabular}

\section{ELISA results}

There was no significant difference in serum IL-2 levels among the 3 groups before 
anesthesia $(P>0.05)$. Compared with before anesthesia, the IL-2 level was reduced at $3 \mathrm{~h}$ and 1 day after the surgery in group $M$ and group $F(P<0.05)$, although there was no significant difference in IL-2 level between before anesthesia and at $3 \mathrm{~h}$ and 3 days after surgery in group $T$ $(P>0.05)$. IL-2 level was slightly increased at 1 day after surgery compared to before anesthesia (Table 2).

\begin{tabular}{|c|c|c|c|c|}
\hline Group & Before anesthesia & $3 \mathrm{~h}$ after the surgery & 1 day after the surgery & 3 days after the surgery \\
\hline Group M & $14 \pm 3$ & $10 \pm 2^{*}$ & $8 \pm 2$ & $11 \pm 1^{*}$ \\
\hline Group T & $14 \pm 3$ & $13 \pm 3$ & $17 \pm 3^{*}$ & $15 \pm 4$ \\
\hline Group F & $13 \pm 1$ & $17 \pm 4^{*}$ & $20 \pm 2^{\star *}$ & $18 \pm 1^{*}$ \\
\hline
\end{tabular}

$\mathrm{N}=40$, means $\pm \mathrm{SD}$ vs before anesthesia. ${ }^{*} \mathrm{P}<0.05$. ${ }^{*} \mathrm{P}<0.01$.

There was no significant difference in serum IL-6 levels among the 3 groups before surgery $(P>0.05)$. Serum IL-6 level was increased in the 3 groups at $3 \mathrm{~h}$ after surgery. IL-6 level reached a maximum at 1 day after surgery. Compared with group $M$ at $1 \mathrm{~h}$ after the surgery, the serum IL-6 level was lower in groups $T$ and $F$; the differences between group $M$ and the other groups were significant $(P<0.05)$. Furthermore, serum IL-6 level was increased to a lower degree in group $F$ than in group $T(P<0.01)$. Moreover, there was a large significant difference in IL-6 level at 3 days after surgery between group $F$ and group $M(P<0.05$, Table 3$)$.

Table 3. Change of IL-6 of three groups vs Group M.

\begin{tabular}{lcccc}
\hline Group & Before anesthesia & $3 \mathrm{~h}$ after the surgery & 1 day after the surgery & 3 days after the surgery \\
\hline Group M & $2 \pm 1$ & $46 \pm 8$ & $81 \pm 10$ & $41 \pm 13$ \\
Group T & $2 \pm 1$ & $39 \pm 7$ & $57 \pm 13^{*}$ & $44 \pm 4$ \\
Group F & $3 \pm 1$ & $33 \pm 2$ & $42 \pm 6^{\star *}$ & $26 \pm 10^{*}$ \\
\hline
\end{tabular}

${ }^{*} \mathrm{P}<0.05,{ }^{* *} \mathrm{P}<0.01$.

Table 4 showed the incidence of adverse effects in the 3 groups. The incidence rate of adverse effects was higher in group $M$ than in the other groups $(P<0.05)$. Additionally, there was a significant difference in the incidence rate of nausea and vomiting between group $F$ and group $M(P<0.05)$.

Table 4. Comparison of adverse effects of three groups vs group M.

\begin{tabular}{lcccc}
\hline Group & Nausea & Vomiting & Pruitus & Respiratory depression \\
\hline Group M & $12(30.0)$ & $16(26.7)$ & $10(25.0)$ & $2(5.0)$ \\
Group T & $8(20.0)^{\star}$ & $6(16.7)^{\star}$ & $0(0)$ & $0(0)$ \\
Group F & $2(5.0)^{* \#}$ & $2(5.0)^{* \#}$ & $0(0)$ & $0(0)$ \\
\hline
\end{tabular}

${ }^{*} \mathrm{P}<0.05 ;$ vs group $\mathrm{T}$, $\mathrm{P}<0.05$.

\section{DISCUSSION}

Perioperative immunosuppression is an important topic for in medicine. Many studies have indicated that postoperative pain is one of the most important factors causing immunosuppression during the perioperative period ( $\mathrm{Li}$ et al., 2013). Rational analgesics may effectively inhibit the stress induced by pain, reduce the incidence of complications, and promote the patient's recovery. However, anesthesia inhibits the stress response induced by operation and pain, and can affect the 
immune system either directly or indirectly. Immune suppression accelerates residual tumor cell growth and metastasis. Typically, immune dysfunction causes infection and septicemia in postoperative patients or patients in the intensive care unit and can lead to infectious shock, multiple organ failure, or even death (Ahlers et al., 2008). Our results indicate that morphine, tramadol, and flurbiprofen axetil all effectively attenuated postoperative pain and decreased the incidence of complications, but opioids may aggravate immune suppression and cause poor prognosis for patients.

Morphine is an opioid analgesic drug first isolated in 1804. Currently, morphine is widely used for clinical analgesic therapy and has very important medical value. However, morphine has immunosuppressive properties and affects the immune system, including $T$ lymphocytes, B lymphocytes, natural killer cells, macrophages, and mononuclear phagocytes (Brown et al., 2012; Abellan et al., 2014). Pain generally leads to immune suppression, which is attenuated by morphine; however, but morphine also has immunosuppressive properties that should not be ignored.

Tramadol is a powerful, centrally acting atypical opioid analgesic that activates the $\mu$-opioid receptor and inhibits serotonin-norepinephrine reuptake, activating the monoamine transmitter systems to inhibit the pain pathway. Thus, tramadol exhibits analgesic action through 2 different pathways (Farquhar-Smith and Gubbay, 2013). Because of the weak affinity of tramadol to the $\mu$-opioid receptor and anti-opioid receptor action, tramadol rarely results in respiration suppression but has good analgesic activity. Additionally, some studies reported that tramadol had adverse effects in the clinic, including nausea, dizziness, vomiting, hypodynamia, hypersomnia sweatiness, and dry mouth (Raffa et al., 2012).

Flurbiprofen axetil injection was first marketed in Japan in July 1992, and the 1-mL emulsion contained $50 \mathrm{mg}$ flurbiprofen axetil. The injection solution was composed of lipid microspheres (LM) and flurbiprofen axetil. The $0.2-\mu \mathrm{m}$ diameter LM is enveloped by the phospholipid membrane. LM affects drug action in 3 ways. First, for targeting, LM envelopes the drug to assemble in inflammatory sites and tumor sites to enhance the effect of the drug. Second, LM controls the release of drug over a long duration. Third, LM can easily cross membranes to promote drug intake and shorten the time necessary for the drug to exhibit its effects (Pergolizzi et al., 2011; Abdel-Aziz et al., 2012). Flurbiprofen axetil is the prodrug of esterified flurbiprofen and alleviates stimulation of the vascular endothelium when injected. Furthermore, flurbiprofen shows powerful anti-inflammatory action in no-steroidal drugs and exhibits few adverse effects; thus, flurbiprofen is widely used in clinical analgesic application. Moreover, numerous animal trails and clinical studies have demonstrated the anti-inflammatory and analgesic action of flurbiprofen, which mainly occurs through inhibiting cyclooxygenase, blocking prostaglandin (PG) production pathways, and reducing the release of inflammatory mediators (Karateev et al., 1998).

IL-2 produced by T cells during an immune response is an important immunoregulatory factor. IL-2 induces the proliferation of T cells, promotes antibody production and proliferation by B cells, and is essential for activation-induced cell death. Moreover, IL-2 enhances natural killer cells activities and promotes interferon secretion (Buchbinder and McDermott, 2014). Therefore, IL-2 plays a central role in the proliferation of immune cells and immune response. In addition, IL-2 level reflects cellular immune activity. When cellular immune function was inhibited, IL-2 level was clearly decreased (Shevach, 2012).

IL- 6 acts as a pro-inflammatory cytokine and is secreted by many lymphocytes, including T lymphocytes, B lymphocytes, and monocytes. IL-6 also plays a role in fighting infection and after trauma; in addition, IL-6 is major factor in the acute phase reaction protein and assembly of inflammatory cells. Furthermore, IL-6 is considered to be an effective index for the trauma stress 
response. High-dose IL-6 generally indicates the presence of an infection, and thus maintaining low levels of IL-6 is beneficial for patient recovery (Kishimoto, 2010).

In this study, morphine significantly decreased postoperative serum IL-2 level. Previous in vitro studies have demonstrated that morphine clearly inhibited the phorbol myristate acetateinduced activation of nuclear factor (NF)- $\mathrm{KB}$, to decrease IL-2 production. NF- $\mathrm{kB}$ is an important transcription factor regulating the cellular response for 150 genes (Kelschenbach et al., 2008). Some studies indicated that morphine affected IL- 6 and tumor necrosis factor- $\alpha$ level by inhibiting $\mathrm{NF}-\mathrm{KB}$ activity. Studies have indicated that morphine has immunosuppressive action, partly by inhibiting NF-KB to mediate the expression of cytokines. Preoperatively and at $3 \mathrm{~h}$ after surgery, there was no significant difference in the level of IL-2 in group M, but IL-2 level was gradually increased at 1 day after surgery $(P<0.01)$, suggesting that tramadol improved immune function and was beneficial for postoperative pain therapy in tumor patients. Compared with group $\mathrm{M}$, group F showed a greater increase in IL-2 level, particularly at 1 day after surgery. This indicates that flurbiprofen axetil had a better effect than tramadol.

Trauma is the main factor that increases IL-6 level. In this study, IL-6 level was increased in all groups, but compared with the morphine group, the IL-6 level was increased to a lower extent in groups $\mathrm{M}$ and F; IL-6 level was lower in group F than in group M. These results indicate that flurbiprofen axetil controls the postoperative inflammatory response and stress response better than tramadol. Moreover, the release of IL-6 was previously associated with increased cyclic AMP and PG improved cellular cyclic AMP level. The analgesic mechanism of non-steroidal antiinflammatory drugs occurred by reducing PG production and inhibiting NF-kB activity to inhibit the release of IL-6 (Wuet al., 2012).

However, the incidence of adverse effects, such as nausea, vomiting, and pruritus, was lower in group $\mathrm{F}$ than in groups $\mathrm{M}$ and $\mathrm{T}$, indicating that flurbiprofen axetil was the safer and more effective analgesic drug.

In conclusion, flurbiprofen axetil, a new non-steroidal analgesic drug, can attenuate the stress response during the perioperative period. Similar to morphine and tramadol, this drug showed good analgesic action and showed few postoperative adverse effects. Thus, we flurbiprofen may be useful for clinical application.

\section{Conflicts of interest}

The authors declare no conflict of interest.

\section{ACKNOWLEDGMENTS}

The authors are pleased to acknowledge the contribution of their colleagues W. Fei, H.W. Liu, S. Guo, L.H. Wang, all of the department of Anesthesiology II, Affiliated Zhongshan Hospital of Dalian University, Dalian, China, to this project. We also acknowledge the useful suggestions on writing and revisions given by the mentor Professor Zhang.

\section{REFERENCES}

Abdel-Aziz AA, Al-Badr AA and Hafez GA (2012). Flurbiprofen. Profiles Drug Subst. Excip. Relat. Methodol. 37: 113-181. Abellan JJ, van Niel JC and Eerdekens M (2014). Tramadol article conclusion troubling. Pain Phys. 17: E418-E419.

Ahlers O, Nachtigall I, Lenze J, Goldmann A, et al. (2008). Intraoperative thoracic epidural anaesthesia attenuates stress- 
induced immunosuppression in patients undergoing major abdominal surgery. Br. J. Anaesth. 101: 781-787.

Atkins MB (2002). Interleukin-2: clinical applications. Semin. Oncol. 29: 12-17.

Brown JN, Ortiz GM, Angel TE, Jacobs JM, et al. (2012). Morphine produces immunosuppressive effects in nonhuman primates at the proteomic and cellular levels. Mol. Cell. Proteomics 11: 605-618.

Buchbinder El and McDermott DF (2014). Interferon, interleukin-2, and other cytokines. Hematol. Oncol. Clin. North Am. 28: 571-583.

Farquhar-Smith P and Gubbay A (2013). Tramadol and acetaminophen combination for chronic non-cancer pain. Expert Opin. Pharmacother. 14: 2297-2304.

Fujii Y and Itakura M (2008). Comparison of lidocaine, metoclopramide, and flurbiprofen axetil for reducing pain on injection of propofol in Japanese adult surgical patients: a prospective, randomized, double-blind, parallel-group, placebo-controlled study. Clin. Ther. 30: 280-286.

Fujii Y and Itakura M (2009). Pretreatment with flurbiprofen axetil, flurbiprofen axetil preceded by venous occlusion, and a mixture offlurbiprofen axetil and propofol in reducing pain on injection of propofol in adult Japanese surgical patients: a prospective, randomized, double-blind, placebo-controlled study. Clin. Ther. 31: 721-727.

Gibson TP (1996). Pharmacokinetics, efficacy, and safety of analgesia with a focus on tramadol HCl. Am. J. Med. 101: 47S-53S

Karateev DE, Nasonova VA and Ivanova MM (1998). Flurbiprofen: pharmacological characteristics and clinical effectiveness. Klin Med. 76: 51-56.

Kelschenbach J, Ninkovic J, Wang J, Krishnan A, et al. (2008). Morphine withdrawal inhibits IL-12 induction in a macrophage cell line through a mechanism that involves cAMP. J. Immunol. 180: 3670-3679.

Kishimoto T (2010). IL-6: from its discovery to clinical applications. Int. Immunol. 22: 347-352.

Li G, Li S, An L and Wang B (2013). Electroacupuncture alleviates intraoperative immunosuppression in patients undergoing supratentorial craniotomy. Acupunct. Med. 31: 51-56.

Mejjad O, Serrie A and Ganry H (2011). Epidemiological data, efficacy and safety of a paracetamol-tramadol fixed combination in the treatment of moderate-to-severe pain. SALZA: a post-marketing study in general practice. Curr. Med. Res. Opin. 27: 1013-1020.

Pahl HL (1999). Activators and target genes of Rel/NF-kappa transcription factors. Oncogene 18: 6853-6866.

Pergolizzi JV Jr, Taylor R Jr and Raffa RB (2011). Extended-release formulations of tramadol in the treatment of chronic pain. Expert Opin. Pharmacother. 12: 1757-1768.

Raffa RB, Buschmann H, Christoph T, Eichenbaum G, et al. (2012). Mechanistic and functional differentiation of tapentadol and tramadol. Expert Opin. Pharmacother. 13: 1437-1449.

Roy S, Ninkovic J, Banerjee S, Charboneau RG, et al. (2011). Opioid drug abuse and modulation of immune function: consequences in the susceptibility to opportunistic infections. J. Neuroimmune Pharmacol. 6: 442-465.

Shevach EM (2012). Application of IL-2 therapy to target T regulatory cell function. Trends Immunol. 33: 626-632.

Wu X1, Lu Y, Dong Y, Zhang Y, et al. (2012). The inhalation anesthetic isoflurane increases levels of proinflammatory TNF- $\alpha$, IL-6, and IL-1ß. Neurobiol. Aging 33: 1364-1378. 